\title{
A Lifting Argument for the Generalized Grigorieff Forcing
}

\author{
Radek Honzík and Jonathan Verner
}

\begin{abstract}
In this short paper, we describe another class of forcing notions which preserve measurability of a large cardinal $\kappa$ from the optimal hypothesis, while adding new unbounded subsets to $\kappa$. In some ways these forcings are closer to the Cohen-type forcings - we show that they are not minimal-but, they share some properties with treelike forcings. We show that they admit fusion-type arguments which allow for a uniform lifting argument.
\end{abstract}

\section{Introduction}

In this short paper, we describe another class of forcing notions which preserve measurability of a large cardinal $\kappa$ from the optimal hypothesis, while adding new unbounded subsets to $\kappa$. A typical application is to force the failure of the generalized continuum hypothesis $(\mathrm{GCH})$ at a measurable cardinal from Assumption 1.1.

Assumption 1.1 There exists $j: V \rightarrow M$ with critical point $\kappa$ and

(i) ${ }^{\kappa} M \subseteq M$;

(ii) there is $f: \kappa \rightarrow \kappa$ such that $j(f)(\kappa)=\kappa^{++}$.

Woodin was the first to force the failure of $\mathrm{GCH}$ at a measurable cardinal from Assumption 1.1 (which is optimal); he used an iteration of the Cohen forcing to achieve this. At the crucial step, when a suitable generic is needed for the Cohen forcing, he solved the problem by modifying an existing generic to fit a certain condition; this is sometimes called "a surgery argument" (see Cummings [3]).

There is an alternative approach, which is more uniform in that the required generic is obtained directly in the current universe. This approach is based on treelike forcings. The first such construction (see Friedman and Thompson [8]) used the generalized Sacks forcing and the accompanying "tuning fork" argument. With the

Received April 11, 2013; accepted November 26, 2013

First published online January 7, 2016

2010 Mathematics Subject Classification: Primary 03E35, 03E55

Keywords: Grigorieff forcing, lifting argument, preserving measurability

(C) 2016 by University of Notre Dame 10.1215/00294527-3459833 


\title{
A Lifting Argument for the Generalized Grigorieff Forcing
}

\author{
Radek Honzík and Jonathan Verner
}

\begin{abstract}
In this short paper, we describe another class of forcing notions which preserve measurability of a large cardinal $\kappa$ from the optimal hypothesis, while adding new unbounded subsets to $\kappa$. In some ways these forcings are closer to the Cohen-type forcings - we show that they are not minimal-but, they share some properties with treelike forcings. We show that they admit fusion-type arguments which allow for a uniform lifting argument.
\end{abstract}

\section{Introduction}

In this short paper, we describe another class of forcing notions which preserve measurability of a large cardinal $\kappa$ from the optimal hypothesis, while adding new unbounded subsets to $\kappa$. A typical application is to force the failure of the generalized continuum hypothesis $(\mathrm{GCH})$ at a measurable cardinal from Assumption 1.1.

Assumption 1.1 There exists $j: V \rightarrow M$ with critical point $\kappa$ and

(i) ${ }^{\kappa} M \subseteq M$;

(ii) there is $f: \kappa \rightarrow \kappa$ such that $j(f)(\kappa)=\kappa^{++}$.

Woodin was the first to force the failure of $\mathrm{GCH}$ at a measurable cardinal from Assumption 1.1 (which is optimal); he used an iteration of the Cohen forcing to achieve this. At the crucial step, when a suitable generic is needed for the Cohen forcing, he solved the problem by modifying an existing generic to fit a certain condition; this is sometimes called "a surgery argument" (see Cummings [3]).

There is an alternative approach, which is more uniform in that the required generic is obtained directly in the current universe. This approach is based on treelike forcings. The first such construction (see Friedman and Thompson [8]) used the generalized Sacks forcing and the accompanying "tuning fork" argument. With the

Received April 11, 2013; accepted November 26, 2013

First published online January 7, 2016

2010 Mathematics Subject Classification: Primary 03E35, 03E55

Keywords: Grigorieff forcing, lifting argument, preserving measurability

(C) 2016 by University of Notre Dame 10.1215/00294527-3459833 


\title{
A Lifting Argument for the Generalized Grigorieff Forcing
}

\author{
Radek Honzík and Jonathan Verner
}

\begin{abstract}
In this short paper, we describe another class of forcing notions which preserve measurability of a large cardinal $\kappa$ from the optimal hypothesis, while adding new unbounded subsets to $\kappa$. In some ways these forcings are closer to the Cohen-type forcings - we show that they are not minimal-but, they share some properties with treelike forcings. We show that they admit fusion-type arguments which allow for a uniform lifting argument.
\end{abstract}

\section{Introduction}

In this short paper, we describe another class of forcing notions which preserve measurability of a large cardinal $\kappa$ from the optimal hypothesis, while adding new unbounded subsets to $\kappa$. A typical application is to force the failure of the generalized continuum hypothesis $(\mathrm{GCH})$ at a measurable cardinal from Assumption 1.1.

Assumption 1.1 There exists $j: V \rightarrow M$ with critical point $\kappa$ and

(i) ${ }^{\kappa} M \subseteq M$;

(ii) there is $f: \kappa \rightarrow \kappa$ such that $j(f)(\kappa)=\kappa^{++}$.

Woodin was the first to force the failure of $\mathrm{GCH}$ at a measurable cardinal from Assumption 1.1 (which is optimal); he used an iteration of the Cohen forcing to achieve this. At the crucial step, when a suitable generic is needed for the Cohen forcing, he solved the problem by modifying an existing generic to fit a certain condition; this is sometimes called "a surgery argument" (see Cummings [3]).

There is an alternative approach, which is more uniform in that the required generic is obtained directly in the current universe. This approach is based on treelike forcings. The first such construction (see Friedman and Thompson [8]) used the generalized Sacks forcing and the accompanying "tuning fork" argument. With the

Received April 11, 2013; accepted November 26, 2013

First published online January 7, 2016

2010 Mathematics Subject Classification: Primary 03E35, 03E55

Keywords: Grigorieff forcing, lifting argument, preserving measurability

(C) 2016 by University of Notre Dame 10.1215/00294527-3459833 


\title{
A Lifting Argument for the Generalized Grigorieff Forcing
}

\author{
Radek Honzík and Jonathan Verner
}

\begin{abstract}
In this short paper, we describe another class of forcing notions which preserve measurability of a large cardinal $\kappa$ from the optimal hypothesis, while adding new unbounded subsets to $\kappa$. In some ways these forcings are closer to the Cohen-type forcings - we show that they are not minimal-but, they share some properties with treelike forcings. We show that they admit fusion-type arguments which allow for a uniform lifting argument.
\end{abstract}

\section{Introduction}

In this short paper, we describe another class of forcing notions which preserve measurability of a large cardinal $\kappa$ from the optimal hypothesis, while adding new unbounded subsets to $\kappa$. A typical application is to force the failure of the generalized continuum hypothesis $(\mathrm{GCH})$ at a measurable cardinal from Assumption 1.1.

Assumption 1.1 There exists $j: V \rightarrow M$ with critical point $\kappa$ and

(i) ${ }^{\kappa} M \subseteq M$;

(ii) there is $f: \kappa \rightarrow \kappa$ such that $j(f)(\kappa)=\kappa^{++}$.

Woodin was the first to force the failure of $\mathrm{GCH}$ at a measurable cardinal from Assumption 1.1 (which is optimal); he used an iteration of the Cohen forcing to achieve this. At the crucial step, when a suitable generic is needed for the Cohen forcing, he solved the problem by modifying an existing generic to fit a certain condition; this is sometimes called "a surgery argument" (see Cummings [3]).

There is an alternative approach, which is more uniform in that the required generic is obtained directly in the current universe. This approach is based on treelike forcings. The first such construction (see Friedman and Thompson [8]) used the generalized Sacks forcing and the accompanying "tuning fork" argument. With the

Received April 11, 2013; accepted November 26, 2013

First published online January 7, 2016

2010 Mathematics Subject Classification: Primary 03E35, 03E55

Keywords: Grigorieff forcing, lifting argument, preserving measurability

(C) 2016 by University of Notre Dame 10.1215/00294527-3459833 


\title{
A Lifting Argument for the Generalized Grigorieff Forcing
}

\author{
Radek Honzík and Jonathan Verner
}

\begin{abstract}
In this short paper, we describe another class of forcing notions which preserve measurability of a large cardinal $\kappa$ from the optimal hypothesis, while adding new unbounded subsets to $\kappa$. In some ways these forcings are closer to the Cohen-type forcings - we show that they are not minimal-but, they share some properties with treelike forcings. We show that they admit fusion-type arguments which allow for a uniform lifting argument.
\end{abstract}

\section{Introduction}

In this short paper, we describe another class of forcing notions which preserve measurability of a large cardinal $\kappa$ from the optimal hypothesis, while adding new unbounded subsets to $\kappa$. A typical application is to force the failure of the generalized continuum hypothesis $(\mathrm{GCH})$ at a measurable cardinal from Assumption 1.1.

Assumption 1.1 There exists $j: V \rightarrow M$ with critical point $\kappa$ and

(i) ${ }^{\kappa} M \subseteq M$;

(ii) there is $f: \kappa \rightarrow \kappa$ such that $j(f)(\kappa)=\kappa^{++}$.

Woodin was the first to force the failure of $\mathrm{GCH}$ at a measurable cardinal from Assumption 1.1 (which is optimal); he used an iteration of the Cohen forcing to achieve this. At the crucial step, when a suitable generic is needed for the Cohen forcing, he solved the problem by modifying an existing generic to fit a certain condition; this is sometimes called "a surgery argument" (see Cummings [3]).

There is an alternative approach, which is more uniform in that the required generic is obtained directly in the current universe. This approach is based on treelike forcings. The first such construction (see Friedman and Thompson [8]) used the generalized Sacks forcing and the accompanying "tuning fork" argument. With the

Received April 11, 2013; accepted November 26, 2013

First published online January 7, 2016

2010 Mathematics Subject Classification: Primary 03E35, 03E55

Keywords: Grigorieff forcing, lifting argument, preserving measurability

(C) 2016 by University of Notre Dame 10.1215/00294527-3459833 


\title{
A Lifting Argument for the Generalized Grigorieff Forcing
}

\author{
Radek Honzík and Jonathan Verner
}

\begin{abstract}
In this short paper, we describe another class of forcing notions which preserve measurability of a large cardinal $\kappa$ from the optimal hypothesis, while adding new unbounded subsets to $\kappa$. In some ways these forcings are closer to the Cohen-type forcings - we show that they are not minimal-but, they share some properties with treelike forcings. We show that they admit fusion-type arguments which allow for a uniform lifting argument.
\end{abstract}

\section{Introduction}

In this short paper, we describe another class of forcing notions which preserve measurability of a large cardinal $\kappa$ from the optimal hypothesis, while adding new unbounded subsets to $\kappa$. A typical application is to force the failure of the generalized continuum hypothesis $(\mathrm{GCH})$ at a measurable cardinal from Assumption 1.1.

Assumption 1.1 There exists $j: V \rightarrow M$ with critical point $\kappa$ and

(i) ${ }^{\kappa} M \subseteq M$;

(ii) there is $f: \kappa \rightarrow \kappa$ such that $j(f)(\kappa)=\kappa^{++}$.

Woodin was the first to force the failure of $\mathrm{GCH}$ at a measurable cardinal from Assumption 1.1 (which is optimal); he used an iteration of the Cohen forcing to achieve this. At the crucial step, when a suitable generic is needed for the Cohen forcing, he solved the problem by modifying an existing generic to fit a certain condition; this is sometimes called "a surgery argument" (see Cummings [3]).

There is an alternative approach, which is more uniform in that the required generic is obtained directly in the current universe. This approach is based on treelike forcings. The first such construction (see Friedman and Thompson [8]) used the generalized Sacks forcing and the accompanying "tuning fork" argument. With the

Received April 11, 2013; accepted November 26, 2013

First published online January 7, 2016

2010 Mathematics Subject Classification: Primary 03E35, 03E55

Keywords: Grigorieff forcing, lifting argument, preserving measurability

(C) 2016 by University of Notre Dame 10.1215/00294527-3459833 


\title{
A Lifting Argument for the Generalized Grigorieff Forcing
}

\author{
Radek Honzík and Jonathan Verner
}

\begin{abstract}
In this short paper, we describe another class of forcing notions which preserve measurability of a large cardinal $\kappa$ from the optimal hypothesis, while adding new unbounded subsets to $\kappa$. In some ways these forcings are closer to the Cohen-type forcings - we show that they are not minimal-but, they share some properties with treelike forcings. We show that they admit fusion-type arguments which allow for a uniform lifting argument.
\end{abstract}

\section{Introduction}

In this short paper, we describe another class of forcing notions which preserve measurability of a large cardinal $\kappa$ from the optimal hypothesis, while adding new unbounded subsets to $\kappa$. A typical application is to force the failure of the generalized continuum hypothesis $(\mathrm{GCH})$ at a measurable cardinal from Assumption 1.1.

Assumption 1.1 There exists $j: V \rightarrow M$ with critical point $\kappa$ and

(i) ${ }^{\kappa} M \subseteq M$;

(ii) there is $f: \kappa \rightarrow \kappa$ such that $j(f)(\kappa)=\kappa^{++}$.

Woodin was the first to force the failure of $\mathrm{GCH}$ at a measurable cardinal from Assumption 1.1 (which is optimal); he used an iteration of the Cohen forcing to achieve this. At the crucial step, when a suitable generic is needed for the Cohen forcing, he solved the problem by modifying an existing generic to fit a certain condition; this is sometimes called "a surgery argument" (see Cummings [3]).

There is an alternative approach, which is more uniform in that the required generic is obtained directly in the current universe. This approach is based on treelike forcings. The first such construction (see Friedman and Thompson [8]) used the generalized Sacks forcing and the accompanying "tuning fork" argument. With the

Received April 11, 2013; accepted November 26, 2013

First published online January 7, 2016

2010 Mathematics Subject Classification: Primary 03E35, 03E55

Keywords: Grigorieff forcing, lifting argument, preserving measurability

(C) 2016 by University of Notre Dame 10.1215/00294527-3459833 


\title{
A Lifting Argument for the Generalized Grigorieff Forcing
}

\author{
Radek Honzík and Jonathan Verner
}

\begin{abstract}
In this short paper, we describe another class of forcing notions which preserve measurability of a large cardinal $\kappa$ from the optimal hypothesis, while adding new unbounded subsets to $\kappa$. In some ways these forcings are closer to the Cohen-type forcings - we show that they are not minimal-but, they share some properties with treelike forcings. We show that they admit fusion-type arguments which allow for a uniform lifting argument.
\end{abstract}

\section{Introduction}

In this short paper, we describe another class of forcing notions which preserve measurability of a large cardinal $\kappa$ from the optimal hypothesis, while adding new unbounded subsets to $\kappa$. A typical application is to force the failure of the generalized continuum hypothesis $(\mathrm{GCH})$ at a measurable cardinal from Assumption 1.1.

Assumption 1.1 There exists $j: V \rightarrow M$ with critical point $\kappa$ and

(i) ${ }^{\kappa} M \subseteq M$;

(ii) there is $f: \kappa \rightarrow \kappa$ such that $j(f)(\kappa)=\kappa^{++}$.

Woodin was the first to force the failure of $\mathrm{GCH}$ at a measurable cardinal from Assumption 1.1 (which is optimal); he used an iteration of the Cohen forcing to achieve this. At the crucial step, when a suitable generic is needed for the Cohen forcing, he solved the problem by modifying an existing generic to fit a certain condition; this is sometimes called "a surgery argument" (see Cummings [3]).

There is an alternative approach, which is more uniform in that the required generic is obtained directly in the current universe. This approach is based on treelike forcings. The first such construction (see Friedman and Thompson [8]) used the generalized Sacks forcing and the accompanying "tuning fork" argument. With the

Received April 11, 2013; accepted November 26, 2013

First published online January 7, 2016

2010 Mathematics Subject Classification: Primary 03E35, 03E55

Keywords: Grigorieff forcing, lifting argument, preserving measurability

(C) 2016 by University of Notre Dame 10.1215/00294527-3459833 


\title{
A Lifting Argument for the Generalized Grigorieff Forcing
}

\author{
Radek Honzík and Jonathan Verner
}

\begin{abstract}
In this short paper, we describe another class of forcing notions which preserve measurability of a large cardinal $\kappa$ from the optimal hypothesis, while adding new unbounded subsets to $\kappa$. In some ways these forcings are closer to the Cohen-type forcings - we show that they are not minimal-but, they share some properties with treelike forcings. We show that they admit fusion-type arguments which allow for a uniform lifting argument.
\end{abstract}

\section{Introduction}

In this short paper, we describe another class of forcing notions which preserve measurability of a large cardinal $\kappa$ from the optimal hypothesis, while adding new unbounded subsets to $\kappa$. A typical application is to force the failure of the generalized continuum hypothesis $(\mathrm{GCH})$ at a measurable cardinal from Assumption 1.1.

Assumption 1.1 There exists $j: V \rightarrow M$ with critical point $\kappa$ and

(i) ${ }^{\kappa} M \subseteq M$;

(ii) there is $f: \kappa \rightarrow \kappa$ such that $j(f)(\kappa)=\kappa^{++}$.

Woodin was the first to force the failure of $\mathrm{GCH}$ at a measurable cardinal from Assumption 1.1 (which is optimal); he used an iteration of the Cohen forcing to achieve this. At the crucial step, when a suitable generic is needed for the Cohen forcing, he solved the problem by modifying an existing generic to fit a certain condition; this is sometimes called "a surgery argument" (see Cummings [3]).

There is an alternative approach, which is more uniform in that the required generic is obtained directly in the current universe. This approach is based on treelike forcings. The first such construction (see Friedman and Thompson [8]) used the generalized Sacks forcing and the accompanying "tuning fork" argument. With the

Received April 11, 2013; accepted November 26, 2013

First published online January 7, 2016

2010 Mathematics Subject Classification: Primary 03E35, 03E55

Keywords: Grigorieff forcing, lifting argument, preserving measurability

(C) 2016 by University of Notre Dame 10.1215/00294527-3459833 


\title{
A Lifting Argument for the Generalized Grigorieff Forcing
}

\author{
Radek Honzík and Jonathan Verner
}

\begin{abstract}
In this short paper, we describe another class of forcing notions which preserve measurability of a large cardinal $\kappa$ from the optimal hypothesis, while adding new unbounded subsets to $\kappa$. In some ways these forcings are closer to the Cohen-type forcings - we show that they are not minimal-but, they share some properties with treelike forcings. We show that they admit fusion-type arguments which allow for a uniform lifting argument.
\end{abstract}

\section{Introduction}

In this short paper, we describe another class of forcing notions which preserve measurability of a large cardinal $\kappa$ from the optimal hypothesis, while adding new unbounded subsets to $\kappa$. A typical application is to force the failure of the generalized continuum hypothesis $(\mathrm{GCH})$ at a measurable cardinal from Assumption 1.1.

Assumption 1.1 There exists $j: V \rightarrow M$ with critical point $\kappa$ and

(i) ${ }^{\kappa} M \subseteq M$;

(ii) there is $f: \kappa \rightarrow \kappa$ such that $j(f)(\kappa)=\kappa^{++}$.

Woodin was the first to force the failure of $\mathrm{GCH}$ at a measurable cardinal from Assumption 1.1 (which is optimal); he used an iteration of the Cohen forcing to achieve this. At the crucial step, when a suitable generic is needed for the Cohen forcing, he solved the problem by modifying an existing generic to fit a certain condition; this is sometimes called "a surgery argument" (see Cummings [3]).

There is an alternative approach, which is more uniform in that the required generic is obtained directly in the current universe. This approach is based on treelike forcings. The first such construction (see Friedman and Thompson [8]) used the generalized Sacks forcing and the accompanying "tuning fork" argument. With the

Received April 11, 2013; accepted November 26, 2013

First published online January 7, 2016

2010 Mathematics Subject Classification: Primary 03E35, 03E55

Keywords: Grigorieff forcing, lifting argument, preserving measurability

(C) 2016 by University of Notre Dame 10.1215/00294527-3459833 


\title{
A Lifting Argument for the Generalized Grigorieff Forcing
}

\author{
Radek Honzík and Jonathan Verner
}

\begin{abstract}
In this short paper, we describe another class of forcing notions which preserve measurability of a large cardinal $\kappa$ from the optimal hypothesis, while adding new unbounded subsets to $\kappa$. In some ways these forcings are closer to the Cohen-type forcings - we show that they are not minimal-but, they share some properties with treelike forcings. We show that they admit fusion-type arguments which allow for a uniform lifting argument.
\end{abstract}

\section{Introduction}

In this short paper, we describe another class of forcing notions which preserve measurability of a large cardinal $\kappa$ from the optimal hypothesis, while adding new unbounded subsets to $\kappa$. A typical application is to force the failure of the generalized continuum hypothesis $(\mathrm{GCH})$ at a measurable cardinal from Assumption 1.1.

Assumption 1.1 There exists $j: V \rightarrow M$ with critical point $\kappa$ and

(i) ${ }^{\kappa} M \subseteq M$;

(ii) there is $f: \kappa \rightarrow \kappa$ such that $j(f)(\kappa)=\kappa^{++}$.

Woodin was the first to force the failure of $\mathrm{GCH}$ at a measurable cardinal from Assumption 1.1 (which is optimal); he used an iteration of the Cohen forcing to achieve this. At the crucial step, when a suitable generic is needed for the Cohen forcing, he solved the problem by modifying an existing generic to fit a certain condition; this is sometimes called "a surgery argument" (see Cummings [3]).

There is an alternative approach, which is more uniform in that the required generic is obtained directly in the current universe. This approach is based on treelike forcings. The first such construction (see Friedman and Thompson [8]) used the generalized Sacks forcing and the accompanying "tuning fork" argument. With the

Received April 11, 2013; accepted November 26, 2013

First published online January 7, 2016

2010 Mathematics Subject Classification: Primary 03E35, 03E55

Keywords: Grigorieff forcing, lifting argument, preserving measurability

(C) 2016 by University of Notre Dame 10.1215/00294527-3459833 